Case

Report

\title{
Pneumothorax Caused by Costal Exostosis
}

\author{
Kentaro Imai, MD, Yasuhiro Suga, MD, Yoshitaka Nagatsuka, MD, Jitsuo Usuda, MD, \\ Tatsuo Ohira, MD, Harubumi Kato, MD, and Norihiko Ikeda, MD
}

\begin{abstract}
Exostosis occurs in the ribs either sporadically or as a manifestation of a genetic disorder known as hereditary multiple exostoses. Costal exostosis may cause chest pain and, on rare occasions, result in hemothorax, pneumothorax, hemopneumothorax, and diaphragmatic or pericardial laceration. We report a case of pneumothorax in a 16year-old boy with exostosis in the right seventh and eighth ribs. He previously received a diagnosis of multiple exostoses and underwent costal resection. The lung and pleura were injured because of long-term friction between the exostoses and the visceral pleura. To the best of our knowledge, this is only the fourth report of pneumothorax caused by costal exostosis. Moreover, all previously reported cases of pneumothorax or hemopneumothorax caused by costal exostosis, including the present case, involved lower lobe injury.
\end{abstract}

Keywords: costal exostosis, rib, pneumothorax, hereditary multiple exostoses

\section{Introduction}

Hereditary multiple exostoses (HME) is an autosomal-dominant condition characterized by exostoses that appear in different parts of the skeleton. Lesions mainly occur in infants and children, and usually cease enlarging at puberty. HME in the ribs (i.e., costal exostosis) is rare and is generally asymptomatic, and about 30 cases of intrathoracic complications have been reported. ${ }^{1,2,3)}$ These complications include hemothorax, pneumothorax, hemopneumothorax, diaphragmatic or pericardial lacerations, and visceral pleural injury. We report a case of pneumothorax caused by long-term costal exostosis. To the best of our knowledge, there are only three previous reports of pneumothorax ${ }^{4,5,6)}$ and one previous report of hemopneumothorax, ${ }^{7)}$ and both conditions were caused by costal exostosis and involved lower lobe injury.

Department of Thoracic Surgery, Tokyo Medical University, Tokyo, Japan

Received: February 28, 2012; Accepted: June 11, 2012 Corresponding author: Kentaro Imai, MD. Department of Thoracic Surgery, Tokyo Medical University, 6-7-1 Nishishinjuku, Shinjuku-ku, Tokyo 160-0023, Japan

Email: kentaro-imai@tea.ocn.ne.jp

(C2014 The Editorial Committee of Annals of Thoracic and Cardiovascular Surgery. All rights reserved.

\section{Case report}

A 16-year-old boy was admitted to our hospital because of right-side pleuritic chest pain. He had no history of recent trauma. He previously received a diagnosis of multiple exostoses, and his family history was significant for multiple exostoses in his grandfather, father, brother, and sister. Chest radiography showed right pneumothorax and a mass arising from the seventh and eighth ribs (Fig. 1). Chest computed tomography (CT) indicated exostosis from the right seventh and eighth ribs anteriorly, with a long, bony spur pointing directly inward to the thoracic cavity (Fig. 2), but CT showed no bulla. Chest drainage was performed for the treatment of his pneumothorax. Four days later, the air leakage stopped. However, as the pneumothorax was considered to be caused by the exostosis, we determined that surgery was necessary to establish the definitive diagnosis and prevent future recurrence. Operation for costal resection was performed by video-assisted thoracoscopic surgery. The first port was made in the anterior axillary line of the sixth intercostal space in which a chest drainage tube was inserted. The second port was placed anterolateral to the fourth intercostal space to which the camera was moved for a better view of the exostosis. The third port was placed posterolateral to the fifth intercostal space. A bony exostosis was found 


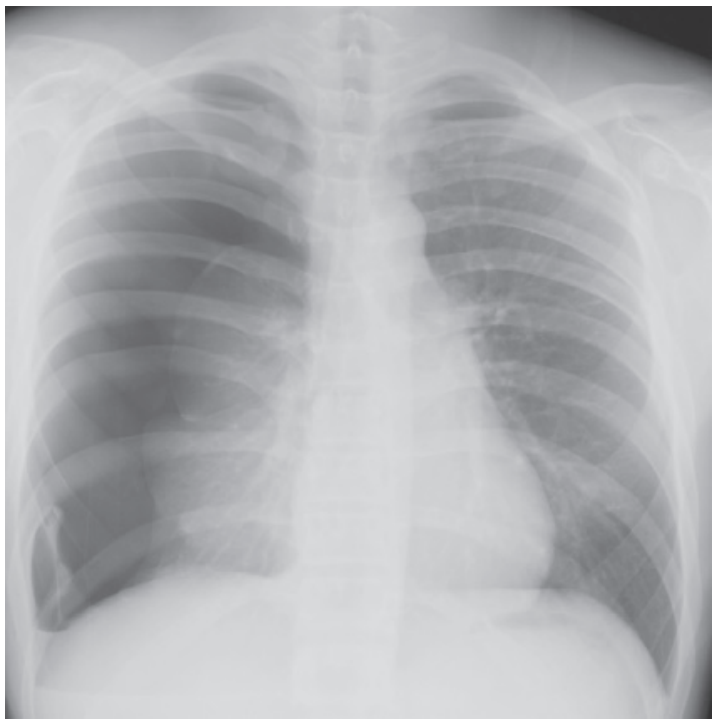

Fig. 1 A chest X-ray film showing pneumothorax and a mass arising from the seventh and eighth ribs.

arising from the posterior aspect of the anterior portion of the seventh and eighth ribs (Fig. 2). There was no obvious clot or source of bleeding, but marked thickness of the visceral pleura of segment eight, facing the exostosis was observed. It was considered that the air leakage originated from this area of thickened visceral pleura. However, the air leakage was stopped intraoperatively, and therefore, we did not perform partial resection of the lung tissue but only resection of part of the rib with the inward-projecting exostosis using a rongeur. His postoperative course was uneventful. A pathological evaluation of an exostosis specimen revealed cartilage, bone, and fibroconnective tissue consistent with findings of exostosis. There was no evidence of malignancy.

\section{Discussion}

HME is an autosomal-dominant condition characterized by multiple exostoses, usually observed in the long tubular bones of the extremities such as the femur, tibia, fibula, humerus, radius, and ulna. Involvement in other bones such as the ribs, scapulae, pelvis, and vertebrae is rare. Solomon ${ }^{8)}$ reported that the ribs were involved in 32 of $76(42 \%)$ patients with exostoses. Despite this relatively high prevalence of exostosis arising from the rib, complications such as hemothorax, pneumothorax, and hemopneumothorax are very rare. About 20 cases of hemothorax as a complication of rib exostosis have been reported, which included injury to the pleura, ${ }^{9)}$ diaphragm, ${ }^{10)}$ and pericardium. ${ }^{11)}$
Three cases of pneumothorax ${ }^{4,5,6)}$ and one case of hemopneumothorax ${ }^{7)}$ as complications of costal exostosis have been reported (Table 1). To the best of our knowledge, the present case is only the fourth report of costal exostosis causing pneumothorax as a result of lung injury. The etiological mechanisms proposed by most authors include laceration to the pleura, diaphragm or pericardium by the relatively sharp margins of an intrathoracic exostosis. In the present case, no obvious source of bleeding or damaged lung tissue was observed and, although there was marked thickening of the visceral pleura located in segment 8 facing the exostosis, neither the pericardium nor the diaphragm was lacerated or punctured. We considered that the local visceral pleural changes were induced by longterm friction between the intrathoracic exostosis and visceral pleura and that spontaneous rupture of the visceral pleura and laceration of the lung may have caused the pneumothorax. The respiratory motion of the lower lobe is greater than that of the middle (in the right lung) or upper lobe. Thus, pneumothorax may easily occur in both the lower lobe and visceral pleura as a result of lung injury caused by exostosis. Previously, in three reports of pneumothorax ${ }^{4,5,6)}$ and in one report of hemopneumothorax, ${ }^{7)}$ all cases involved lower lobe injury caused by exostosis, although one case was not described in detail. Rib exostosis can be difficult to visualize on standard chest X-ray films. Chest $\mathrm{CT}$ is usually more useful in establishing the diagnosis and confirming associated intrathoracic abnormalities. Costal exostosis in HME can be much easier to diagnose if there is a significant familial history, and because of the frequent involvement of characteristic deformities resulting from the deficient growth of involved bones. ${ }^{12)}$ Patients with HME should be examined using CT to detect the presence of intrathoracic exostosis and if detected should be closely followed up.

\section{Acknowledgements}

We are indebted to Mr. Roderick J. Turner, Associate Professor Edward F. Barroga and Professor J. Patrick Barron, Chairman of the Department of International Medical Communications of Tokyo Medical University, for their editorial review of the English manuscript.

\section{Disclosure Statement}

The authors declare that they have no conflicts of interest associated with this study. 
Table 1 Previous reports in the literature of pneumothorax and hemopneumothorax caused by costal exostosis

\begin{tabular}{|c|c|c|c|c|c|c|c|}
\hline Case & Author & Sex & Age (years) & Exostosis location & Diagnosis & Injured region & Treatment \\
\hline 1 & Pollitzer, et al. & M & 27 & left 8 th rib & pneumothorax & left lower lobe & $\begin{array}{l}\text { rib removal, sterile } \\
\text { talc applied to } \\
\text { the visceral pleura }\end{array}$ \\
\hline 2 & Suzuki, et al. & M & 21 & right 7 th rib & pneumothorax & right lower lobe & $\begin{array}{c}\text { rib removal, suturing } \\
\text { of injured } \\
\text { visceral pleura }\end{array}$ \\
\hline 3 & Bini, et al. & M & 36 & right 9 th rib & hemopneumothorax & right lower lobe & $\begin{array}{c}\text { rib removal, suturing } \\
\text { of injured } \\
\text { visceral pleura }\end{array}$ \\
\hline 4 & Khosla, et al. & M & 17 & left 5 th rib & pneumothorax & not described & rib removal \\
\hline
\end{tabular}
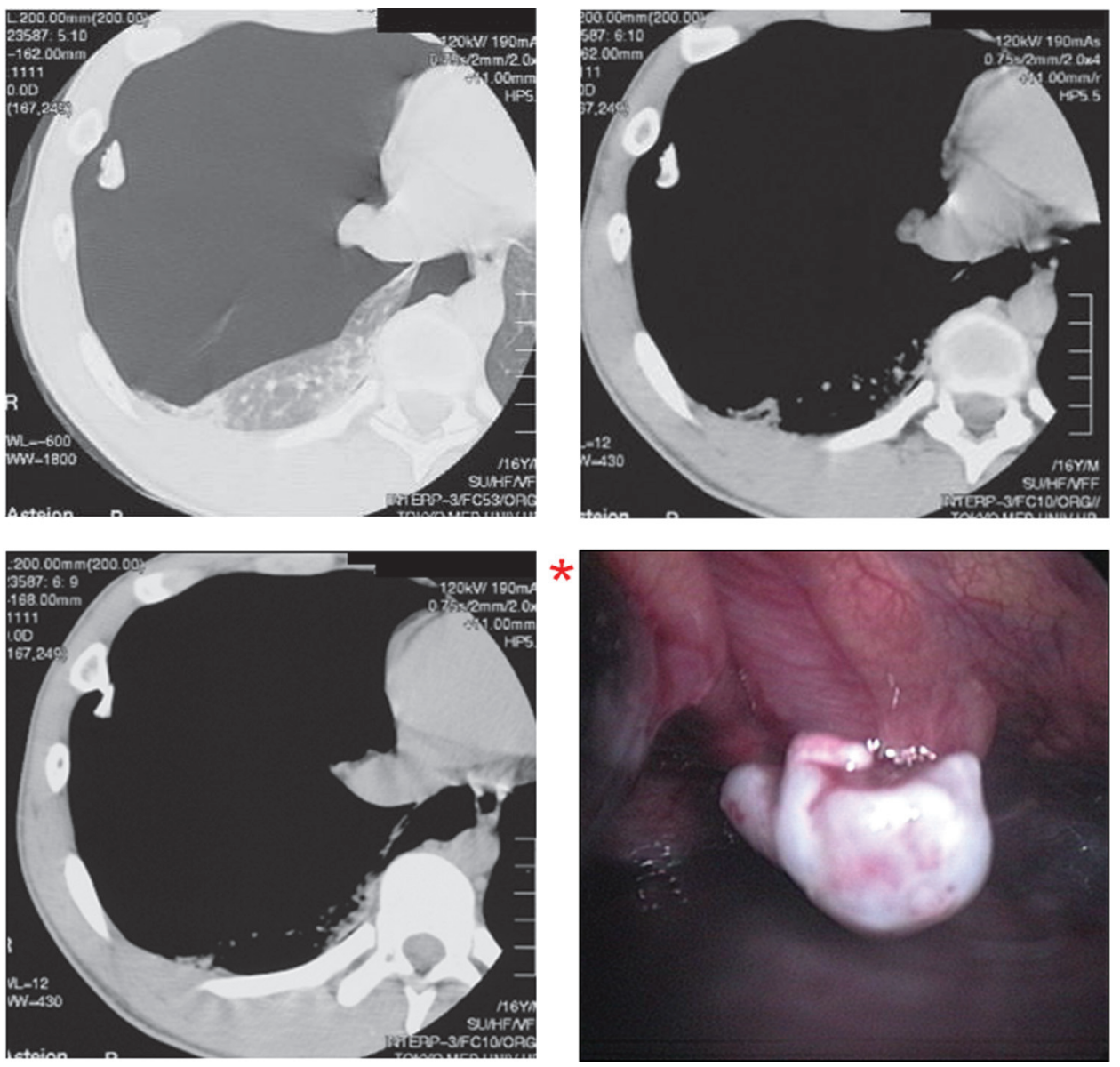

Fig. 2 A computed tomography scan showing exostosis from the right seventh and eighth ribs and a collapsed right lung. ${ }^{*}$ Intraoperative findings of the right seventh and eighth rib exostosis.

\section{References}

1) Cowles RA, Rowe DH, Arkovitz MS. Hereditary multiple exostoses of the ribs: an unusual cause of hemothorax and pericardial effusion. J Pediatr Surg 2005; 40: 1197-200.

2) Uchida K, Kurihara Y, Sekiguchi S, et al. Spontaneous haemothorax caused by costal exostosis. Eur Respir J 1997; 10: 735-6.
3) Reynolds JR, Morgan E. Haemothorax caused by a solitary costal exostosis. Thorax 1990; 45: 68-9.

4) Khosla A, Parry RL. Costal osteochondroma causing pneumothorax in an adolescent: a case report and review of the literature. J Pediatr Surg 2010; 45: 2250-3.

5) Suzuki T, Hori G, Yoshimatsu T, et al. A case of pneumothorax in a patient with costal exostosis. 
Nihon Kyobu Geka Gakkai Zasshi 1991; 39: 1077-80. (in Japanese)

6) Pollitzer RC, Harrell GT, Postlethwait RW. Recurrent pneumothorax associated with hereditary deforming chondrodysplasia; report of a case apparently due to puncture of the lung by an exostosis of a rib. N C Med J 1952; 13: 668-73.

7) Bini A, Grazia M, Stella F, et al. Acute massive haemopneumothorax due to solitary costal exostosis. Interact Cardiovasc Thorac Surg 2003; 2: 614-5.

8) Solomon L. Hereditary multiple exostoses. J Bone Joint Surg 1963; 45: 292-304.
9) Takata K, Suzuki K, Kurosaki Y. Spontaneous hemothorax in hereditary multiple exostosis involving the ribs. Radiat Med 2008; 26: 39-41.

10) Simansky DA, Paley M, Werczberger A, et al. Exostosis of a rib causing laceration of the diaphragm: diagnosis and management. Ann Thorac Surg 1997; 63: 856-7.

11) Buchan KG, Zamvar V, Mandana KM, et al. Juxtacardiac costal osteochondroma presenting as recurrent haemothorax. Eur J Cardiothorac Surg 2001; 20: 208-10.

12) Castells L, Comas P, González A, et al. Case report: haemothorax in hereditary multiple exostosis. $\mathrm{Br} \mathrm{J}$ Radiol 1993; 66: 269-70. 